\title{
Modeling HIV Vaccines in Brazil: Assessing the Impact of a Future HIV Vaccine on Reducing New Infections, Mortality and Number of People Receiving ARV
}

\author{
Maria Goretti P. Fonseca ${ }^{1 *}$, Steven Forsythe ${ }^{2}$, Alexandre Menezes ${ }^{3}$, Shilpa Vuthoori ${ }^{4}$, Cristina Possas ${ }^{5}$, \\ Valdiléa Veloso ${ }^{1}$, Francisca de Fátima Lucena ${ }^{6}$, John Stover ${ }^{2}$
}

1 Instituto de Pesquisa Clínica Evandro Chagas, Fundação Oswaldo Cruz, Rio de Janeiro, Rio de Janeiro, Brazil, 2 Futures Institute, Glastonbury, Connecticut, United States of America, 3 International AIDS Vaccine Initiative, New York, New York, United States of America, 4 Rabin Strategic Partners, New York, New York, United States of America, $\mathbf{5}$ Departamento de DST, AIDS e Hepatites Virais, Ministério da Saúde, Brasília, Federal District, Brazil, 6 Ministério do Desenvolvimento Social e Combate à Fome, Brasília, Federal District, Brazil

\begin{abstract}
Background: The AIDS epidemic in Brazil remains concentrated in populations with high vulnerability to HIV infection, and the development of an HIV vaccine could make an important contribution to prevention. This study modeled the HIV epidemic and estimated the potential impact of an HIV vaccine on the number of new infections, deaths due to AIDS and the number of people receiving ARV treatment, under various scenarios.

Methods and Findings: The historical HIV prevalence was modeled using Spectrum and projections were made from 2010 to 2050 to study the impact of an HIV vaccine with $40 \%$ to $70 \%$ efficacy, and $80 \%$ coverage of adult population, specific groups such as MSM, IDU, commercial sex workers and their partners, and 15 year olds. The possibility of disinhibition after vaccination, neglecting medium- and high-risk groups, and a disease-modifying vaccine were also considered. The number of new infections and deaths were reduced by $73 \%$ and $30 \%$, respectively, by 2050 , when $80 \%$ of adult population aged $15-$ 49 was vaccinated with a $40 \%$ efficacy vaccine. Vaccinating medium- and high-risk groups reduced new infections by $52 \%$ and deaths by $21 \%$. A vaccine with $70 \%$ efficacy produced a great decline in new infections and deaths. Neglecting medium- and high-risk population groups as well as disinhibition of vaccinated population reduced the impact or even increased the number of new infections. Disease-modifying vaccine also contributed to reducing AIDS deaths, the need for ART and new HIV infections.
\end{abstract}

Conclusions: Even in a country with a concentrated epidemic and high levels of ARV coverage, such as Brazil, moderate efficacy vaccines as part of a comprehensive package of treatment and prevention could have a major impact on preventing new HIV infections and AIDS deaths, as well as reducing the number of people on ARV. Targeted vaccination strategies may be highly effective and cost-beneficial.

Citation: Fonseca MGP, Forsythe S, Menezes A, Vuthoori S, Possas C, et al. (2010) Modeling HIV Vaccines in Brazil: Assessing the Impact of a Future HIV Vaccine on Reducing New Infections, Mortality and Number of People Receiving ARV. PLoS ONE 5(7): e11736. doi:10.1371/journal.pone.0011736

Editor: Landon Myer, University of Cape Town, South Africa

Received March 29, 2010; Accepted June 29, 2010; Published July 23, 2010

Copyright: (c) 2010 Fonseca et al. This is an open-access article distributed under the terms of the Creative Commons Attribution License, which permits unrestricted use, distribution, and reproduction in any medium, provided the original author and source are credited.

Funding: The authors acknowledge the International AIDS Vaccine Initiative for financially supporting this study as part of its Impact Modeling Project, which was made possible by the generous support of the American people through the United States for International Development (USAID). The contents are the responsibility of the authors and do not necessarily reflect the views of USAID or the United States Government. The funding sources had no involvement in the study design, in the collection, analysis, and interpretation of data, in the writing of the report, or in the decision to submit the paper for publication.

Competing Interests: The authors have declared that no competing interests exist.

* E-mail: gorettifonseca@fiocruz.br

\section{Introduction}

After 30 years the AIDS pandemic continues to compromise the health, quality of life and productivity of populations, and significantly affects economies as well as societal and family structures. Although the epidemic in Brazil remains concentrated amongst populations with increased vulnerability to HIV infection [1], more than 500,000 AIDS cases and 200,000 AIDS deaths have been reported by 2008 [2].

To address the challenge of continued HIV transmission as well as increasing treatment needs, the Brazilian government has prioritized prevention efforts [3-4], and has implemented a broad treatment and care program throughout the five geographical regions in the country. The treatment program is based on technical guidelines determined by a national expert committee, which ensures that all patients living with HIV and AIDS in need of antiretroviral (ARV) treatment have access to state-of-the-art care, including options for patients resistant to first and second line regimens [5].

While much has been achieved with the adoption of existing preventive measures and the introduction of potent drugs that severely reduce viral load, there is still a pressing need to develop additional prevention options, such as HIV vaccines, to drastically reduce the number of new infections. A number of studies have been published that involve the application of mathematical modeling to questions of the potential impact of an HIV vaccine 
Table 1. Parameter Values used to Simulate the Brazilian HIV Epidemic.

\begin{tabular}{llll}
\hline Parameter & Value & Source \\
\hline $\begin{array}{l}\text { Relative infectiousness compared to } \\
\text { asymptomatic stage }\end{array}$ & & Pilcher [25] \\
$\quad$ Primary stage & 8 & \\
$\quad$ Symptomatic stage & 4 & \\
$\begin{array}{l}\text { Probability of HIV transmission per act, } \\
\text { heterosexual contact }\end{array}$ & 0.0007 & Powers [26] \\
$\begin{array}{l}\text { Transmission multiplier for MSM contacts } \\
\text { Duration of (in years): }\end{array}$ & 2.6 & Vittinghoff [27] \\
\hline Asymptomatic stage & & Fitted \\
\multicolumn{1}{c}{ Symptomatic stage } & 10 & \\
Condom efficacy & 3 & \\
Annual survival on ART & 0.8 & Weller [28] \\
\hline doi:10.1371/journal.pone.0011736.t001 & 0.95 & \\
\hline
\end{tabular}

on HIV incidence or prevalence in a variety of epidemic settings (see [6] for a review of this literature). These studies have generally shown that even a moderate-efficacy vaccine could have a significant impact in reducing new HIV infections and could represent a vital strategy to control the epidemic. Recent mathematical model studies have shown that a moderately effective vaccine given to the adult population could reduce future HIV prevalence in low- and middle- income countries by $30 \%$ to $35 \%$ in 10 to 15 years [7-8].

Considering the specificities of the AIDS epidemic in Brazil, this study was undertaken to model the Brazilian epidemic and to estimate the potential impact of an HIV vaccine on the number of new infections, deaths due to AIDS and the number of people receiving antiretroviral (ARV) treatment, under various scenarios.

\section{Methods}

The Spectrum HIV Vaccine Module [9] was used to model the historical prevalence of HIV in Brazil from 1975 to 2010 and the impact of an HIV vaccine on the future course of the epidemic. Details of the model structure and key assumptions are available elsewhere [10]. The model divides the sexually active population aged 15-49 by sex and risk group. Although some infections occur at ages above 50 , the percentage is small, less than $10 \%$, and most behavioral indicators refer to the 15-49 age group. The risk groups are injecting drug users, men who have sex with men, high risk heterosexuals (female sex workers and their male clients), medium risk heterosexuals (men and women with more than one partner in the last year) and low risk heterosexuals (men and women with only one partner in the last year). The probability of acquiring a new HIV infection is determined by characteristics of the index person (number of partners), the partner (HIV status, stage of infection, ART use), and the partnership (sex acts per partner, condom use, STI prevalence, heterosexual or MSM contact, male circumcision status). Infected persons progress through a primary stage of infection with high infectivity, an asymptomatic stage with low infectivity, a symptomatic stage with high infectivity, to AIDS death. Infectivity is reduced by ARV use. The model includes a component to estimate the effects of prevention interventions on key behaviors (condom use, number of partners, unsafe injecting behavior) based on a summary of the impact literature [11].

The model was set up for Brazil using behavioral data from surveys [12-22], epidemiological [2,23] and ART data [24] from the Brazilian Ministry of Health $(\mathrm{MOH})$. We calibrated the model to HIV prevalence data by risk group and validated it by comparing the estimated AIDS mortality, HIV prevalence and the number of people receiving ARV with respective data from the $\mathrm{MOH}[2,23-$ 24]. Although there are no official estimates of incidence to which the model results can be compared, the number of new infections estimate is a consequence of matching official estimates of mortality and prevalence. The parameter [25-28] and behavioral values used [12-22] are shown in Tables 1 and 2.

The HIV vaccine module can be used to examine the effects of vaccines with difference characteristics: reduction in susceptibility, disease progression and/or infectiousness, duration of effectiveness and take or degree type of action. Vaccination programs can be defined in terms of coverage over time of the entire adult population or targeted coverage for specific population groups.

The impacts of vaccines with different characteristics and the impacts of different implementation strategies were explored under a variety of different assumptions. In this paper, we present the results for a vaccine introduced in 2015 and with $80 \%$ coverage reached by 2020 , with duration of 20 years. We assume that an HIV test is not a requirement for vaccination. ART coverage is nearly universal in Brazil today and we assumed that would continue in all projections.

Table 2. Current Behaviors used to Simulate the Brazilian HIV Epidemic, 2007.

\begin{tabular}{|c|c|c|c|c|c|c|}
\hline & \multicolumn{6}{|l|}{ Risk groups } \\
\hline & Not sexually active & Low & Medium & High & MSM & IDU \\
\hline \multicolumn{7}{|c|}{ Population distribution (\%) } \\
\hline Males & 6.5 & 66.5 & 18.2 & 4.5 & 3.5 & 0.8 \\
\hline Females & 15.3 & 78.8 & 4.6 & 0.9 & - & 0.4 \\
\hline Condom use (\%) & & 7 & 16 & 52 & 80 & \\
\hline \multicolumn{7}{|l|}{ Partners \# } \\
\hline Males & & 1 & 3 & 10 & 4 & \\
\hline Females & & 1 & 3 & 75 & - & \\
\hline Sex acts per partner \# & & 70 & 40 & 5 & 25 & \\
\hline
\end{tabular}

Source [12-22].

doi:10.1371/journal.pone.0011736.t002 
The vaccine scenarios explored the potential impact of a $40 \%$ and $70 \%$ effective preventive "take" vaccine, which reduces both susceptibility and transmission. A "take" vaccine is one that achieves an efficacy of $50 \%$, for example, by completely protecting $50 \%$ of those vaccinated and providing no protection for the other $50 \%$, as opposed to a "degree" vaccine that achieves $50 \%$ efficacy by reducing susceptibility by $50 \%$ in all those vaccinated.

This study also looked at the potential impact of diverse vaccination strategies, including vaccinating the general adult population, aged 15-49 (including high risk populations); vaccinating 15-year-old adolescents; and vaccinating medium- and high-risk groups, defined as men who have sex with men (MSM), injecting drug users (IDU), and commercial sex workers and their partners (considered here as medium- and high-risk behavior heterosexuals).

In addition, a vaccine scenario that models the potential impact of a disease-modifying vaccine, defined as a vaccine that reduces infectiousness and delays progression to the development of AIDS, and consequently slows the progression to AIDS death, or, in other words, that would not affect individual susceptibility to HIV infection but instead reduces infectiousness and lengthens the amount of time spent in the asymptomatic phase by reducing viral load, was explored.

To assess the potential effect of behavioral disinhibition on the trajectory of Brazil's epidemic in the presence of an AIDS vaccine, vaccine impact scenarios were explored in which a $50 \%$ decline in condom use was considered either immediately after vaccine introduction or more gradually over time. To further explore the effectiveness of targeted vaccination strategies, the study also modeled the impact of failure to reach the medium- and high-risk population groups, by vaccinating $20 \%$ of these groups while reaching $80 \%$ of low-risk adult population aged 15-49.

\section{Results}

The model consistency can be seen in Figures 1 and 2. As can be observed, the progression of AIDS deaths over time adequately reflects the history of the epidemic in Brazil (Figure 1). Spectrum estimated 182,562 cumulative AIDS deaths for those aged 15-49, $12.3 \%$ more deaths when compared with 162,545 deaths reported by the Brazilian Mortality Information System for the period 1985-2007. When comparing the number of people receiving ARV, the Spectrum estimates were also similar to the official data, which report about 190,000 on ARV by the end of 2008 (Figure 2). The HIV prevalence estimated by Spectrum was slightly lower $(0.40 \%)$ in 2004 , when compared to a $68 \%$ confidence interval from the official estimate $(0.44 \%-0.77 \%)$.

In order to capture the longer- term impact of an HIV vaccine, we simulated the epidemic to 2050. Spectrum projects 1.1 million new infections and 468,000 deaths from 2010 to 2050, keeping HIV prevalence relatively stable around 0.42 in the absence of a vaccine or changes in preventive measures and in patient treatment coverage.

\section{Scenarios to study the impact of an HIV vaccine in the Brazilian epidemic}

Compared with a baseline scenario with no vaccine, vaccinating $80 \%$ of the adult population with a $40 \%$ efficacy vaccine reduced the cumulative number of new HIV infections from 2020-2050 by $73 \%$ (Figure 3 ) and AIDS deaths by $30 \%$ (Figure 4). Vaccinating the medium- and high-risk behavior population resulted in an estimated $52 \%$ reduction in new infections (Figure 3 ) and 21\% reduction in AIDS deaths (Figure 4). However, the smallest impact was observed when vaccination strategies targeted only adolescents.

A $70 \%$ effective vaccine given to the general population would result in a significantly greater impact, interfering dramatically in the Brazilian epidemic by reducing the number of new infections from 2020 to 2050 by $92 \%$. Even a targeted strategy of vaccinating only medium- and high-risk behavior population groups with a $70 \%$ effective vaccine would still produce dramatic results, reducing new infections by $74 \%$.

\section{A disease-modifying vaccine scenario}

When the general population was vaccinated with a diseasemodifying vaccine, the cumulative number of new infections was reduced by $46 \%$ (Figure 5) and the number of deaths had a $30 \%$ reduction (Figure 6) from 2020 to 2050. A lower impact was observed when only specific groups were targeted for vaccination.

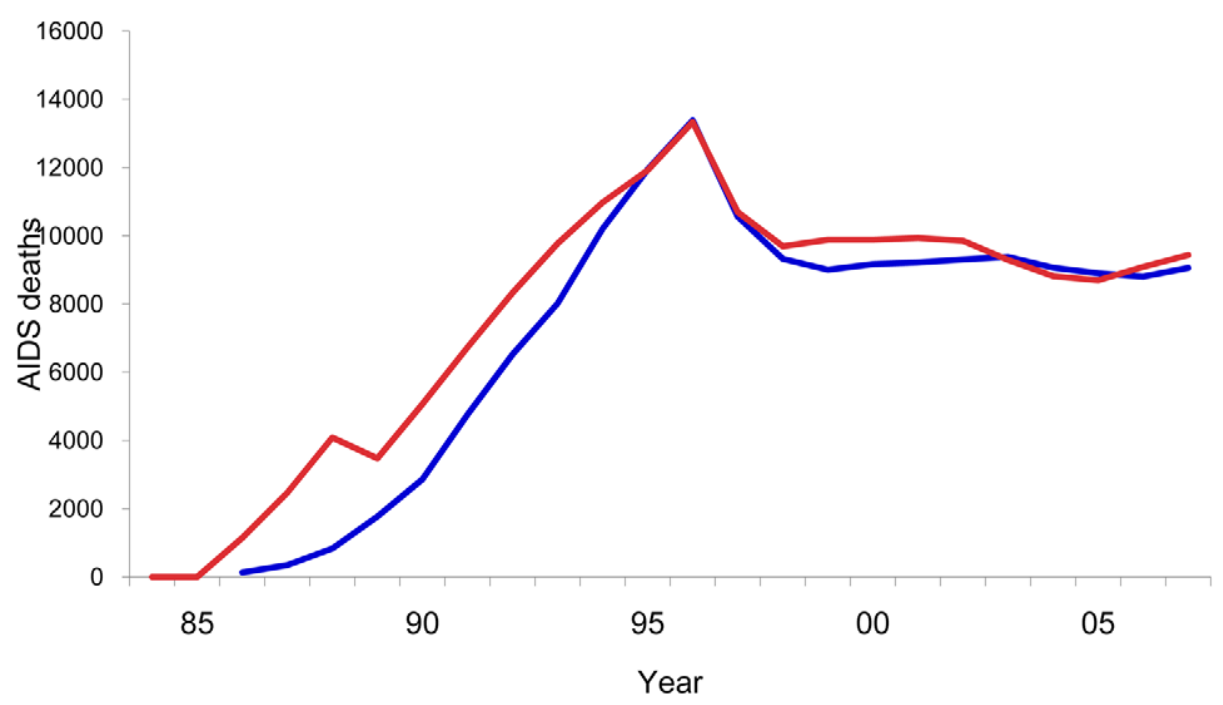

Figure 1. AIDS deaths model fit. Number of AIDS deaths and model fit. Brazil, 1985-2007. The blue line, deaths for age group 15-49 as reported by the Brazilian National Mortality Information System; the red line, Spectrum estimates of number of AIDS deaths in the same age group. doi:10.1371/journal.pone.0011736.g001 


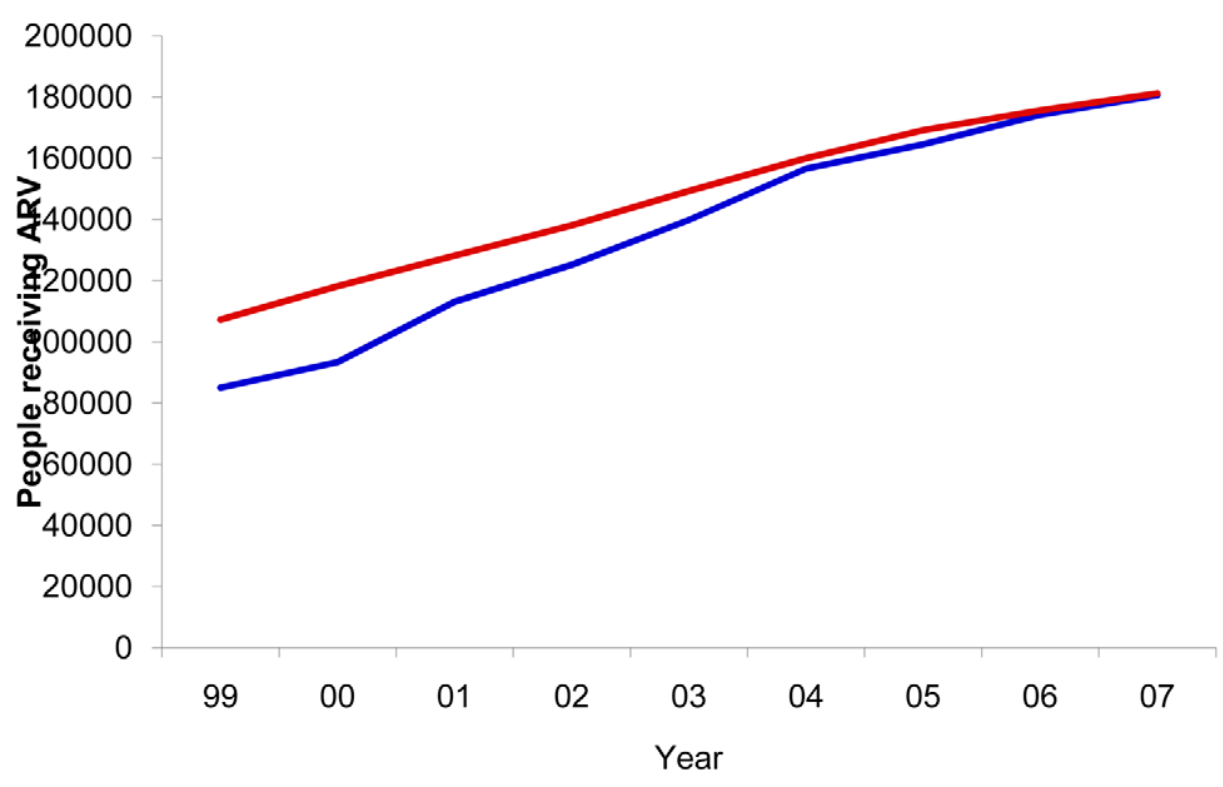

Figure 2. ARV patients and model fit. Number of patients receiving ARV and model fit. Brazil, 1999-2007. The blue line, number of patients of all age reported by the Brazilian STD, AIDS and Viral Hepatitis Department; the red line, Spectrum estimates of number of patients receiving ARV aged $15-49$.

doi:10.1371/journal.pone.0011736.g002

Disease-modifying vaccines were also shown to be effective in reducing the number of patients receiving $\mathrm{ARV}$, although not as effective as preventive vaccines (Figure 7).

\section{Assessment of the relative impact of targeted vaccination strategies}

With the understanding that public health officials will consider vaccination strategies not only on the basis of their overall impact, but also taking into account dose availability at the time of licensing, cost of the vaccine and highest impact in relation to investment, the study explored how the different vaccine strategies compared in terms of impact and the number of doses required for each strategy. Table 3 summarizes the vaccine efficacy, the percentage reduction, the cumulative number of new HIV infections averted, the number of doses required and the number of HIV infections averted per 100,000 vaccinations, according to population vaccinated from 2020 to 2050 , for both preventive and disease-modifying vaccines. With $80 \%$ coverage, the highest reduction in the number of new infections is reached if the general population aged 15-49 is vaccinated, although requiring a very high number of vaccine doses. On the other hand, the better result in terms of number of new infections averted per 100,000 doses is obtained when medium- and high-risk behavior population groups are prioritized for vaccination, with 4 times more

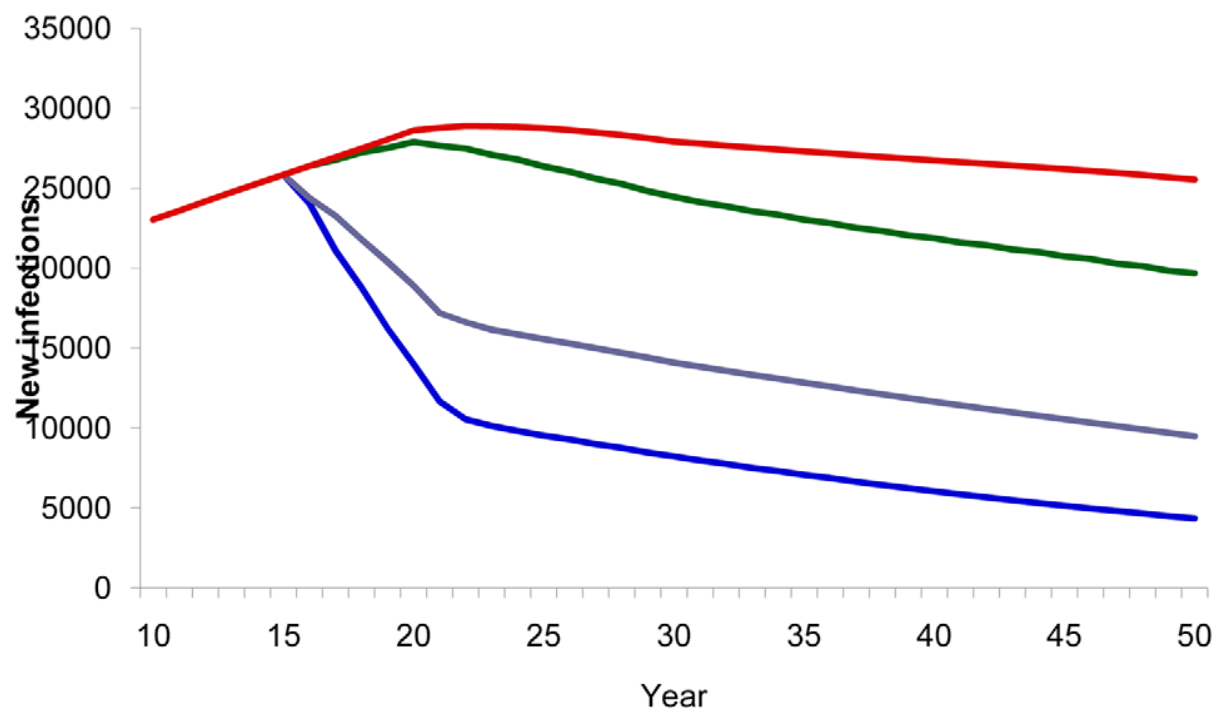

Figure 3. HIV vaccine impact on new infections. Number of estimated new HIV infections according to population vaccinated by year. Red line, baseline projection in the absence of a vaccine; green line, vaccination of adolescents (15 years old); purple line, vaccination of specific population: MSM, IDU, medium- and high-risk heterosexuals; blue line, vaccination of adults aged 15-49.

doi:10.1371/journal.pone.0011736.g003 


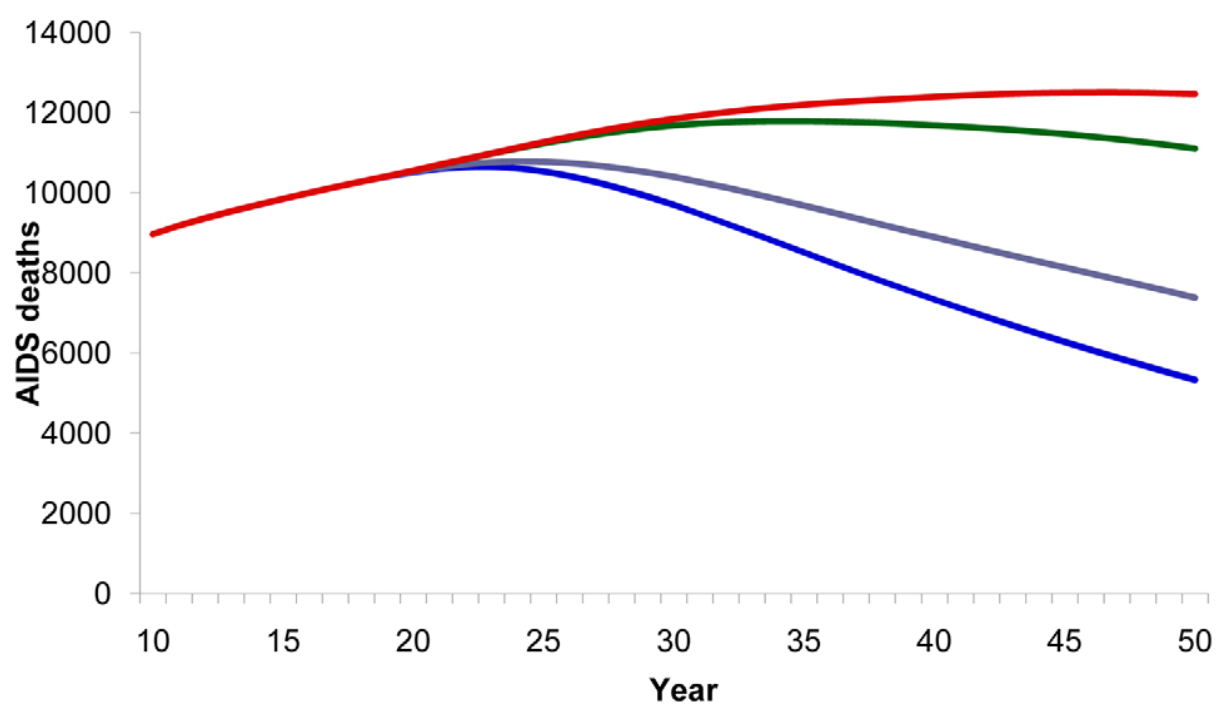

Figure 4. HIV vaccine impact on AIDS deaths. Number of estimated AIDS deaths according to population vaccinated by year. Red line, baseline projection in the absence of a vaccine; green line, vaccination of adolescents (15 years old); purple line, vaccination of specific population: MSM, IDU, medium- and high-risk heterosexuals; blue line, vaccination of adults aged 15-49.

doi:10.1371/journal.pone.0011736.g004

infections averted compared to vaccinating the general adult population (Table 3).

\section{Behavior reversal (disinhibition) and failure to reach medium- and high-risk behavior population groups}

A few additional scenarios were considered to assess the relevance of factors that may reduce or interfere with the impact of a vaccine, such as lack of effective education and counseling of vaccine recipients, leading to behavior disinhibition, and failure to reach and vaccinate populations at higher risk. Two scenarios were considered to look into the potential importance of behavior disinhibition. In the first scenario, the number of new infections was reduced by $42 \%$ ( $42 \%$ instead of $73 \%$ ) when the general population was vaccinated with a low-efficacy vaccine and gradually reduced condom use. In the second analysis regarding behavior dishinibition, the study observed an $8.5 \%$ increase in the cumulated number of new HIV infections when $50 \%$ of the medium- and high-risk behavior vaccinated population immediately stopped using condoms (Figure 8). This remains true even with a more effective vaccine (Figure 9).

The analysis shows that the most effective strategies are those that target vaccinations to medium- and high-risk groups, as seen by the number of infections averted per 100,000 people vaccinated in Table 3. To demonstrate the importance of ensuring adequate coverage of high-risk individuals, the study explored the impact of reaching $80 \%$ vaccination coverage among the low-risk adult

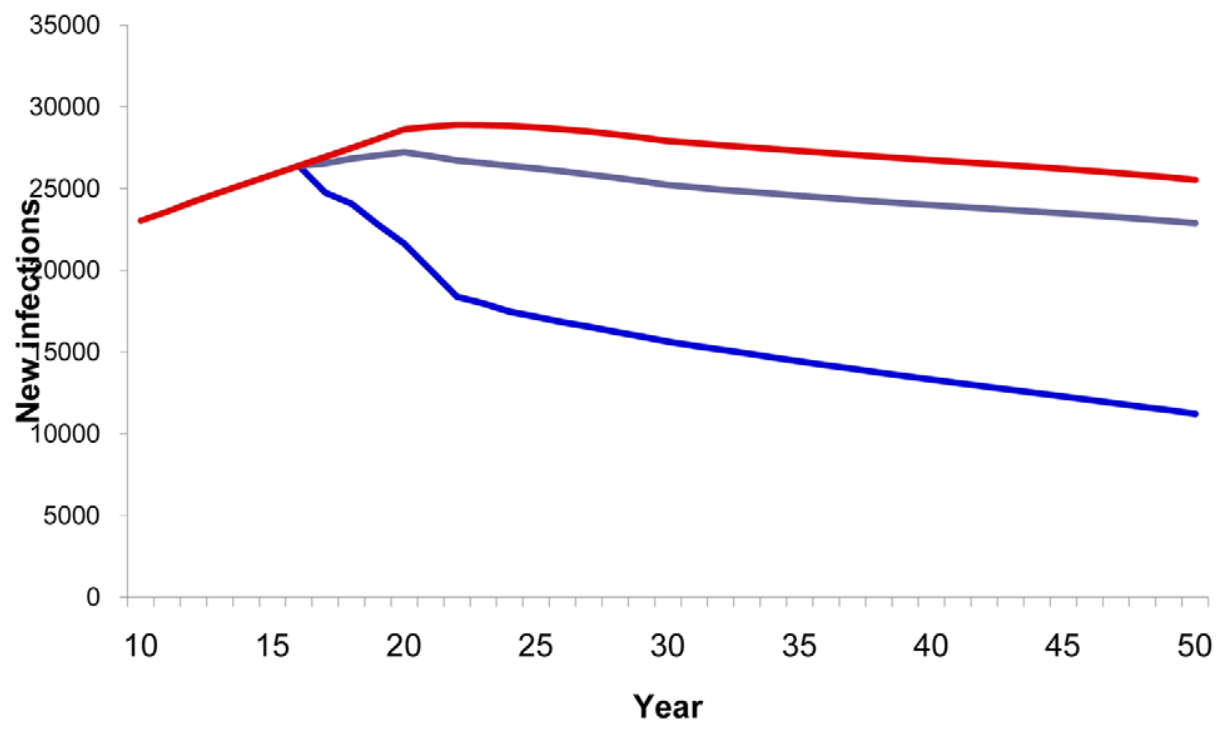

Figures 5. Impact of disease-modifying vaccine on number of new HIV infections. Number of new HIV infections according to population vaccinated with a disease-modifying vaccine. Red line, baseline projection in the absence of a vaccine; blue line, vaccination of adults aged 15-49; purple line, vaccination of specific population: MSM, IDU, medium- and high-risk heterosexuals.

doi:10.1371/journal.pone.0011736.g005 


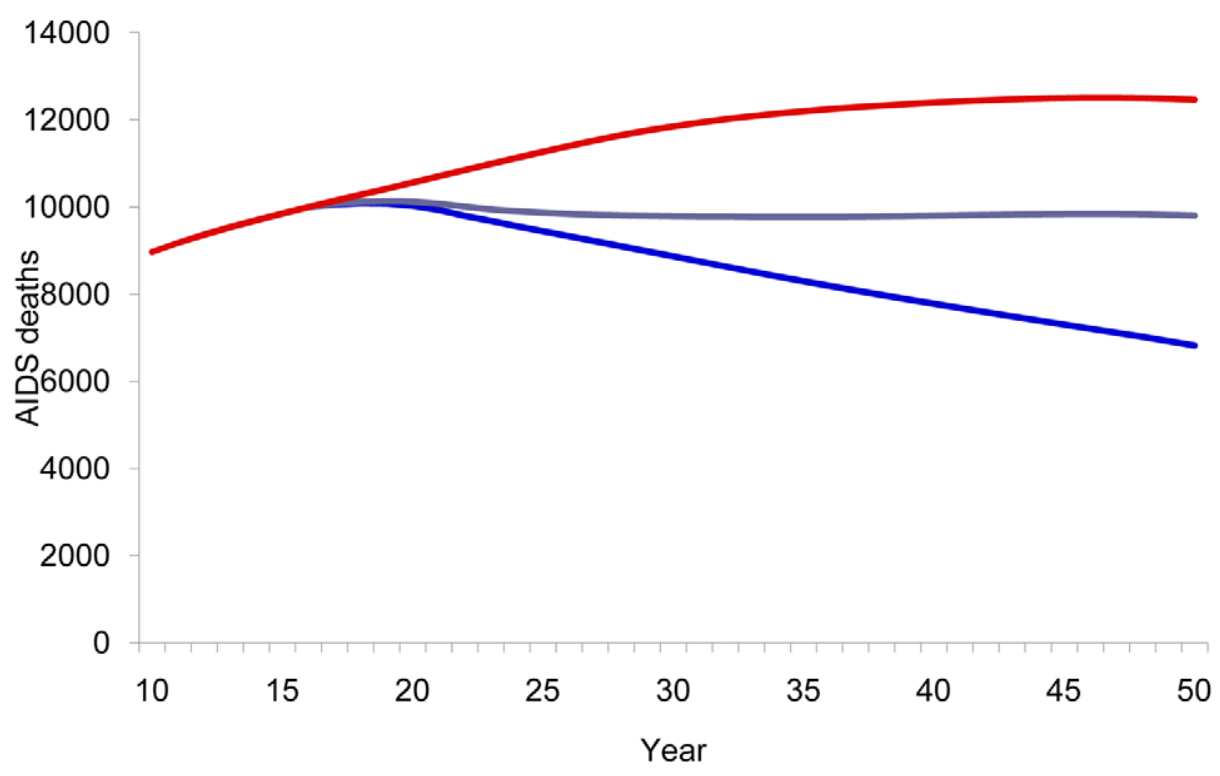

Figure 6. Impact of disease-modifying vaccine on number of AIDS deaths. Number of AIDS deaths according to population vaccinated with a disease-modifying vaccine. Red line, baseline projection in the absence of a vaccine; blue line, vaccination of adults aged 15-49; purple line, vaccination of specific population: MSM, IDU, medium- and high-risk heterosexuals.

doi:10.1371/journal.pone.0011736.g006

population, but only including $20 \%$ of the medium- and high-risk behavior groups in the vaccinated cohort. This scenario was shown to prevent $29 \%$ fewer new HIV infections $(52 \%$ compared to $73 \%$ ) when compared to a vaccination strategy that also includes the medium- and high-risk population groups (Figure 10).

\section{Discussion}

Although the development of an effective HIV vaccine is still years away, modeling studies such as this one are relevant and critical to preparing governments for adopting appropriate policies when a vaccine becomes available $[8,29]$ or as a vaccine candidate advances into later stages of clinical testing. Mathematical modeling and computer simulation are powerful tools for health policy evaluation, policy dialogue, and advocacy efforts [8-9,30-31].

Spectrum was able to effectively model the Brazilian epidemic, producing projections of the number of new infections, AIDS deaths and people needing ARV based on various vaccine profiles and vaccination strategies. This exercise has shown that, even in a country like Brazil with a concentrated epidemic and ample availability of treatment and services for people with HIV and AIDS, a partially-effective vaccine could significantly reduce the number of new HIV infections and the future need for treatment, as well as the deaths caused by AIDS.

A unique aspect of this modeling application is that the epidemiological model was validated by comparing estimates of AIDS deaths for the period 1985 to 2007 with those reported by

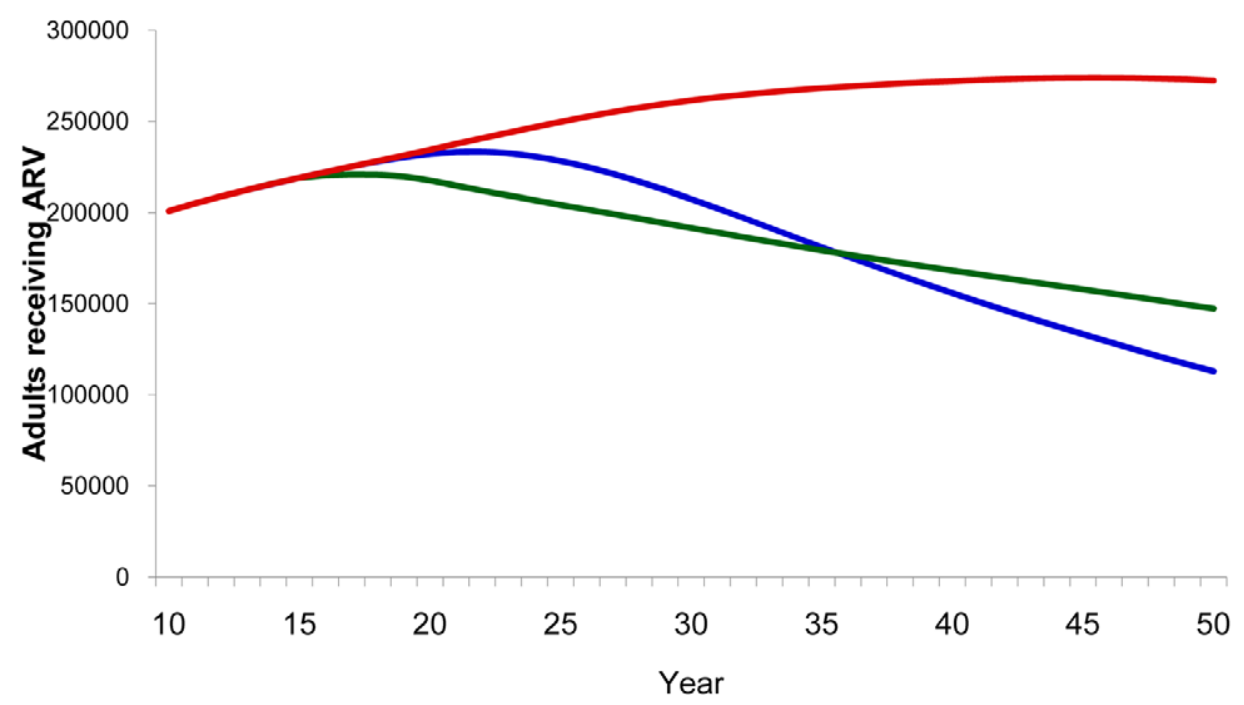

Figure 7. Patients on ARV. Estimated number of patients receiving ARV according to type of vaccine by year. Red line, baseline projection in the absence of a vaccine; blue line, $40 \%$ efficacy preventive vaccine; green line, $100 \%$ disease-modifying vaccine with $40 \%$ reduction in infectiousness. doi:10.1371/journal.pone.0011736.g007 
Table 3. Vaccination Strategies.

\begin{tabular}{|c|c|c|c|c|c|}
\hline Population to be vaccinated & $\begin{array}{l}\text { New HIV } \\
\text { infections (\#) }\end{array}$ & $\begin{array}{l}\text { Reduction in new } \\
\text { infections }(\%)^{*}\end{array}$ & $\begin{array}{l}\text { Infections } \\
\text { averted (\#) }\end{array}$ & $\begin{array}{l}\text { Doses of } \\
\text { vaccine required } \\
\text { (per } 1 \text { million) }\end{array}$ & $\begin{array}{l}\text { Infections averted per } \\
100,000 \text { vaccinations (\#) }\end{array}$ \\
\hline \multicolumn{6}{|c|}{ Preventive Vaccine/Vaccine efficacy (\%) } \\
\hline \multicolumn{6}{|l|}{ Adults (aged 15-49) } \\
\hline 40 & 230203 & 72.8 & 617647 & 241 & 256 \\
\hline 70 & 72369 & 91.5 & 775481 & 241 & 322 \\
\hline \multicolumn{6}{|l|}{ Medium- \& High-risk behavior groups } \\
\hline 40 & 405639 & 52.2 & 442211 & 43 & 1023 \\
\hline 70 & 222754 & 73.7 & 625096 & 43 & 1447 \\
\hline \multicolumn{6}{|l|}{ 15-year-olds } \\
\hline 40 & 725383 & 14.4 & 122467 & 43 & 283 \\
\hline 70 & 643471 & 24.1 & 204379 & 43 & 472 \\
\hline \multicolumn{6}{|c|}{ Adults, excluding medium- and high- risk behavior groups } \\
\hline 40 & 408880 & 51.8 & 438970 & 208 & 211 \\
\hline 70 & 214547 & 74.7 & 633303 & 208 & 304 \\
\hline \multicolumn{6}{|l|}{ Disease-Modifying Vaccine } \\
\hline Adults (aged 15-49) & 458773 & 45.9 & 389077 & 241 & 161 \\
\hline Medium- \& High-risk behavior groups & 767485 & 9.5 & 80365 & 43 & 186 \\
\hline
\end{tabular}

Vaccine efficacy, cumulative number of new HIV infections, percentage reduction in relation to a no vaccine scenario, cumulative number of new HIV infections averted, number of doses required, and number of infections averted per 100,000 population vaccinated according to population to be vaccinated and type of vaccine (preventive or disease modifying).

*Compared to No Vaccine Scenario, where 847,850 new HIV infections would occur from 2020 to 2050 in the absence of vaccination.

doi:10.1371/journal.pone.0011736.t003

the vital registration system. This validation also included the rapid decline in AIDS deaths after the introduction of ART. It is possible that all AIDS deaths were not reported and that Spectrum has overestimated the AIDS deaths in Brazil. However, several studies have shown that the Brazilian Mortality System is quite reliable in terms of completeness and accuracy, as well as in relation to cause of death [32-33].

In the base projection, the annual number of new infections increases somewhat from 2010 to 2020 largely due to an increase among IDU, required to maintain constant prevalence after a period of declining prevalence among that group. A projection of a constant number of new HIV infections among IDU may be equally likely but, in either case, this assumption has little effect on the major conclusions. Also, the base projection without a vaccine shows a slight rise in the number of AIDS deaths. This is due to increasing mortality among those who have been on ART since the rapid scale-up of ART coverage in the 1990s.

Even though the model replicates the historical trends in Brazil, there is no guarantee that the model is correct in all aspects. With so many factors influencing model behavior it is likely that there are other sets of parameters and behavioral values that would fit the historical trends equally well. The impact of vaccines might be different with these other configurations. Advanced modeling techniques can be used to explore the impacts in the complete parameter space [34-35] but we have not done that here.

The impact analysis focuses on the period after 2020 when $80 \%$ coverage is reached and the impact of the vaccine is most significant. This relatively high coverage rate was deemed realistic by the project's steering committee - formed by Brazilian advisors with thorough understanding of the national epidemic - because of Brazil's relatively strong health infrastructure and successful coverage of existing health technologies, such as currently licensed vaccines and ARVs.

Efficacies of $40 \%$ and $70 \%$ were chose to represent a plausible range of likely vaccines that might be used in national programs. While a vaccine with efficacy greater than $70 \%$ would certainly be desirable, the difficulties to date in developing an effective vaccine indicates that the first vaccines to become available are not likely to provide perfect protection. Vaccines with efficacies below $30-40 \%$ are not likely to become approved for widespread use given the possibilities of risk compensation leading to perverse results. The recently completed RV144 vaccine trial indicated efficacy of around $30 \%$ [36]. These results are being used to guide future research rather than supporting immediate introduction of the tested vaccine.

A vaccine with $40 \%$ effectiveness given to $80 \%$ of the population would reduce susceptibility by $32 \%$ ( $40 \%$ times $80 \%)$, but the longterm effect is much larger, $72 \%$ from 2020 to 2050 . The reason is that the reduction in susceptibility leads to fewer new infections which, in turn leads to lower prevalence. Thus in the future the susceptible population not only has reduced susceptibility to infection but also faces a reduced risk of encountering and infected partner. Over the long-term these secondary effects can be as large or larger than the reduction in susceptibility.

The analysis presented here has shown that vaccinating the adult population achieved the largest reduction of new HIV infections and deaths compared to other strategies. However, vaccinating specific population groups, such as those with higherrisk behavior, produced a greater impact with fewer vaccine doses required, which is important to note in resource-constrained environments. Through the use of targeted strategies, a significant proportion of the groups at increased risk for HIV can be reached with a smaller number of vaccinations than those required for vaccinating the adult population. For this reason, it is likely that a 


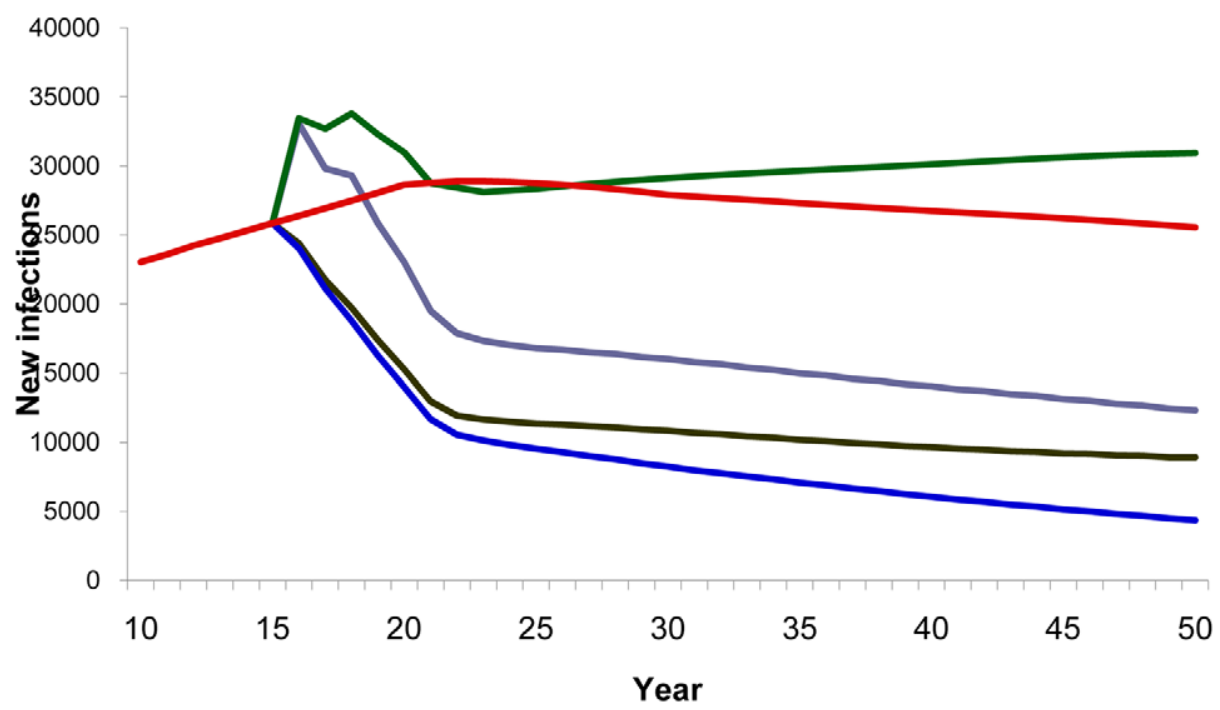

Figure 8. Impact of disinhibition with $\mathbf{4 0 \%}$ efficacy vaccine. Number of new HIV infections estimates with a $40 \%$ efficacy vaccine and presence of disinhibition. Red line, baseline projection in the absence of a vaccine; blue line, vaccination of adults aged 15-49; brown line, vaccination of adults aged 15-49 with gradual disinhibition; purple line, vaccination of adults aged 15-49 with instantaneous disinhibition; green line, vaccination of specific population (MSM, IDU, medium- and high-risk heterosexuals) with instantaneous disinhibition.

doi:10.1371/journal.pone.0011736.g008

targeted vaccination strategy focusing on higher incidence groups would likely be more cost-effective, with a higher number of infections averted per vaccination. However, it is crucial that vaccination strategies for the adult population be designed to reach groups at greater risk, ensuring that these groups have equal access to the vaccine. Vaccinating only individuals at lower risk for HIV would significantly diminish the impact of the vaccine, compromising the goal of controlling the epidemic.

One concern with regards to implementing partially efficacious vaccines is the issue of behavioral disinhibition, which occurs when vaccinated individuals believe they are protected from HIV infection and therefore increase the behaviors that put them at greater risk of exposure to HIV [6]. Several studies have described how behavioral reversals could mitigate the gains from vaccination or even produce perverse outcomes where the use of the vaccine results in more infections [8,37-39]. This study reinforces those findings. Even in a country with a concentrated epidemic, disinhibition will be a great challenge with the potential to negate the impact of a partially efficacious HIV vaccine. Efforts should be made to keep adherence to preventive measures at a high level, especially among populations at greater risk of infection, even when an HIV vaccine becomes available.

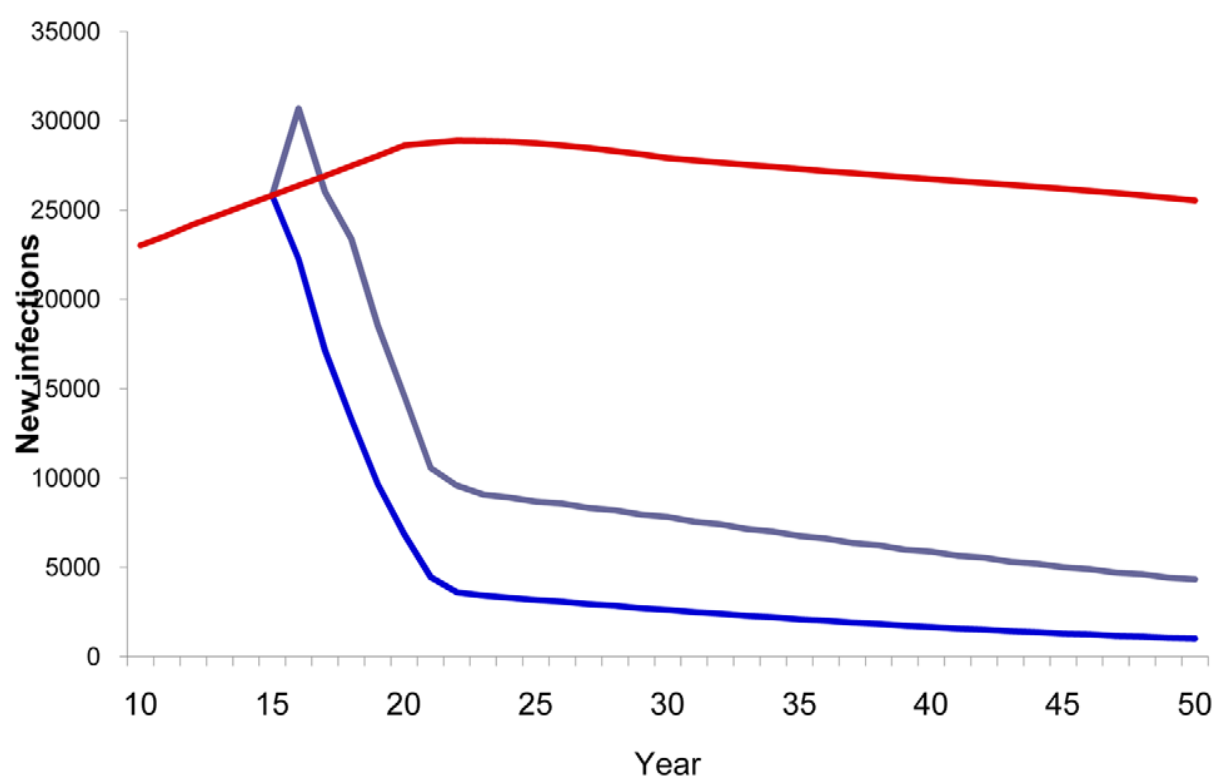

Figure 9. Impact of disinhibition with $\mathbf{7 0} \%$ efficacy vaccine. Number of new HIV infections estimates with a $70 \%$ efficacy vaccine and presence of disinhibition. Red line, baseline projection in the absence of a vaccine; blue line, vaccination of adults aged 15-49; purple line, vaccination of adults aged 15-49 with instantaneous disinhibition.

doi:10.1371/journal.pone.0011736.g009 


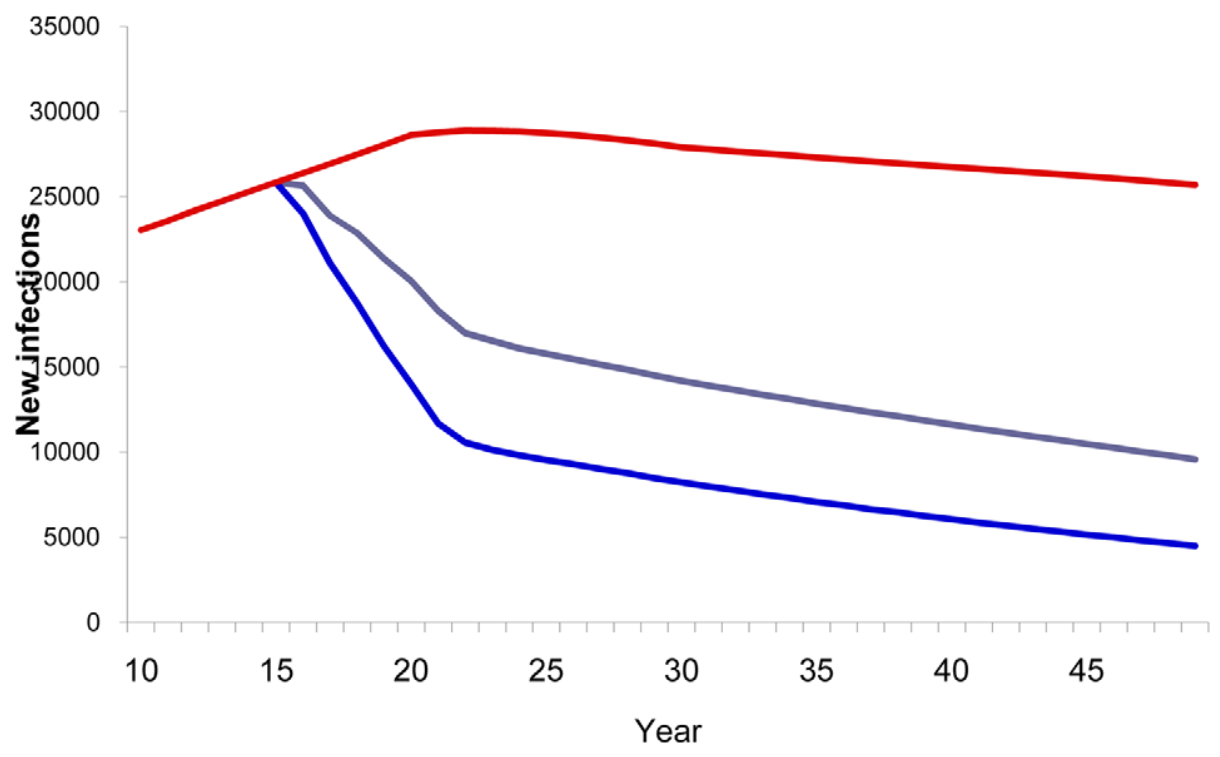

Figure 10. Failure to reach medium- and high-risk behavior groups. Estimated number of new HIV infections with a $40 \%$ efficacy vaccine according to vaccine coverage (\%) of population targeted. Red line, baseline projection in the absence of a vaccine; blue line, $80 \%$ coverage of adults aged 15-49; purple line, $80 \%$ coverage of low-risk-adults aged 15-49 and 20\% coverage of specific population: MSM, IDU, medium- and high-risk heterosexuals.

doi:10.1371/journal.pone.0011736.g010

Although vaccines that partially prevent infection may produce a greater impact on the course of the epidemic, it is important to stress the potential benefits of a disease-modifying vaccine. This modeling exercise has demonstrated that such a vaccine could reduce AIDS deaths and also substantially decreases the demand for ARV immediately after introduction. A disease-modifying vaccine would also reduce infectiousness, further contributing to control the epidemic [40-42]. Without a reduction in infectiousness, disease-modifying vaccines would likely lead to more infections [40-41,43-44] but fewer deaths [40,44]. This study did not investigate the impact of a vaccine in either the absence of or reduction in ARV coverage because Brazil has a governmental policy to provide treatment to all in need.

The potential impact of even a moderate-efficacy vaccine as indicated in this study can also provide a powerful argument for the relevance of continued investment in research and development towards a vaccine as it presents evidence of how efforts to control HIV would dramatically benefit from it, even in the context of concentrated epidemics. For middle-income countries such as Brazil that have well established scientific and technological capacity, the results of this study, which highlight the relevance of a vaccine for the Brazilian epidemic, as well as others

\section{References}

1. Fonseca MGP, Bastos FI (2007) Twenty-five years of the AIDS epidemic in Brazil: principal epidemiological findings, 1980-2005. Cad Saude Publica 23(Sup 3): S333-S344.

2. Brasil. Ministério da Saúde. Secretaria de Vigilância em Saúde. Departamento de DST, Aids e Hepatites Virais (2008) Boletim Epidemiológico AIDS DST V(01). Available: http://www.aids.gov.br/data/Pages/LUMIS9A49113DPTBRIE. htm. Accessed 2010 Jun 23.

3. Brasil. Ministério da Saúde. Secretaria de Vigilância em Saúde. Programa Nacional de DST e Aids (2007) Recomendações para Profilaxia da Transmissão Vertical do HIV e Terapia Antirretroviral em Gestantes - 2006. Série Manuais no 46 .

4. Brasil. Ministério da Saúde. Secretaria de Vigilância em Saúde. Programa Nacional de DST e Aids (2008) Plano Nacional de Enfrentamento da Epidemia de Aids e das DST entre gays, HSH e travestis. 2007. Available: http://www.aids. gov.br/main.asp? View $=\% 7 \mathrm{BE} 62 \mathrm{~A} 8511 \% 2 \mathrm{D} 7150 \% 2 \mathrm{D} 4615 \% 2 \mathrm{D} 9 \mathrm{BFA} \% 2 \mathrm{D} 10 \mathrm{FD}$ $\mathrm{C} 4 \mathrm{~F} 4 \mathrm{E} 642 \%$ 7D $\&$ Team $=$ \& params $=$ sLetraFim $=\mathrm{Q} \% 3$ BsLetraIni $=\mathrm{P} \% 3 \mathrm{~B} \&$ UIPart with same characteristics, should stimulate the implementation of more effective policies to promote and facilitate research in this field. Moreover, conclusions of the study related to the relative impact of vaccination strategies may prove particularly relevant in informing and accelerating discussions around vaccine introduction and delivery encouraging rapid adoption of a future product.

\section{Acknowledgments}

We would like to thank Gerson Pereira, from the Ministry of Health; Paula M Luz, Francisco Bastos and Maeve Mello from Oswaldo Cruz Foundation; and Angélica Miranda from Universidade Federal do Espírito Santo, for their helpful advice; and Wilson Lee from the International AIDS Vaccine Initiative for his writing assistance.

\section{Author Contributions}

Conceived and designed the experiments: MGPF SF AM SSV CP VGV FdFdAL JS. Analyzed the data: MGPF SF FdFdAL JS. Contributed reagents/materials/analysis tools: MGPF. Wrote the paper: MGPF SF AM SSV CP VGV FdFdAL JS. Participated in the discussion of the results: AM SSV CP VGV FdFdAL.

$\mathrm{UID}=\%$ 7BC002F320\%2D7F48\%2D4FD5\%2D9036\%2D124927BAF21E $\% 7 \mathrm{D}$. Accessed 2010 Jun 22.

5. Brasil. Ministério da Saúde. Secretaria de Vigilância em Saúde. Departamento de DST, Aids e Hepatites Virais (2010) Recomendações para Terapia Antirretroviral em Adultos Infectados pelo HIV - 2008 - Suplemento II. Available: http://www.aids.gov. $\mathrm{br} / \mathrm{main}$.asp ? View $=\% 7 \mathrm{BE} 62 \mathrm{~A} 8511 \% 2 \mathrm{D} 7150 \% 2 \mathrm{D} 4615 \% 2 \mathrm{D} 9 \mathrm{BFA} \% 2 \mathrm{D} 10 \mathrm{FDC} 4$ F4E642\%7D\&Team $=$ \&params $=$ sLetraFim $=S \% 3$ BsLetraIni $=\mathrm{R} \% 3 \mathrm{~B} \&$ UIPartUI $\mathrm{D}=\%$ 7BC002F320\%2D7F48\%2D4FD5\%2D9036\%2D124927BAF21E \%7D. Accessed 2010 Jun 22.

6. International AIDS Vaccine Initiative (2005) Modeling the Impact of AIDS Vaccines: A Review of the Literature. Policy Research Working Paper \#5.

7. Stover J, Garnett GP, Seitz S, Forsythe S (2002) The epidemiological impact of an HIV/AIDS vaccine in developing countries. Policy Research Working Paper 2811. The World Bank. March.

8. Anderson KM, Owens DK, Vardas E, Gray GE, McIntyre JA, et al. (2007) Predicting the impact of a partially effective HIV vaccine and subsequent risk 
behavior change on the heterosexual HIV epidemic in low- and middle-income countries. J Acquir Defic Syndr 46: 78-90.

9. Stover J, Johnson P, Zaba B, Zwahlen M, Dabis F, et al. (2008) The Spectrum projection package: improvements in estimating mortality, ART needs, PMTCT impact and uncertainty bounds. Sex Transm Infect 84(Suppl I): i24-i30. The Spectrum model can be downloaded from www.FuturesInstitute.org. The projection files can be provided by the author upon request.

10. Stover J, Bollinger L, Hecht R, Williams C, Roca E (2007) The Impact of an AIDS Vaccine in Developing Countries: A New Model and Initial Results. Health Affairs 26: 1147-1158.

11. Bollinger LA (2008) How can we calculate the "E" in "CEA"? AIDS 22 (suppl 1): S51-S57.

12. Hacker MA, Leite IC, Renton A, Torres TG, Gracie R, et al. (2006) Reconstructing the AIDS epidemic among injection drug users in Brazil. Cad Saude Publica 22: 751-60.

13. Szwarcwald CL, Carvalho MF, Barbosa Junior A, Barreira D, Speranza FA, et al. (2005) Temporal trends of HIV-related risk behavior among Brazilian military conscripts, 1997-2002. Clinics 60: 367-74.

14. Ministério da Saúde. Secretaria de Políticas de Saúde. Coorção Nacional de DST e AIDS (2000) Comportamento Sexual da População Brasileira e Percepções do HIV/Aids. Série Avaliação No. 4, PN DST e Aids, Brasília, 2000.

15. Ministério da Saúde. Secretaria de Políticas de Saúde. Coorção Nacional de DST e AIDS (2002) Pesquisa entre os Conscritos do Exército Brasileiro, 19962000: Retratos do comportamento de risco do jovem brasileiro à infecção pelo HIV. Série Estudos Pesquisas e Avaliação no 2. Brasília, Ministério da Saúde, CN DST/Aids, 2002.

16. Sociedade Civil Bem-Estar Familiar no Brasil BEMFAM (1996) Programa de Pesquisas de Demografia e Saúde (DHS) Macro International Inc, 1997. Brasil Pesquisa Nacional Sobre Demografia e Saúde 1996.

17. Pires ICP, Miranda AEB (1998) Prevalência e Fatores Correlatos de Infecção pelo HIV e Sífilis em Prostitutas Atendidas em Centro de Referência DST/ AIDS RBGO, 20(3): 151-154.

18. Ministério da Saúde. Secretaria de Políticas de Saúde. Programa Nacional de DST e AIDS (2004) Avaliação da Efetividade das ações de Prevenção dirigidas às Profissionais do Sexo, em Três Regiões Brasileiras. Série Estudos, Pesquisas e Avaliação, n 7 , Ministério da Saúde, 2004

19. Ministério da Saúde. Secretaria de Políticas de Saúde. Coordção Nacional de DST e Aids (2001) Projeto Ajude-Brasil: Avaliação Epidemiológica dos Usuários de Drogas Injetáveis dos projetos de redução de danos apoiados pela CN-DST/ AIDS. Série Avaliação no 6, Brasília, Ministério da Saúde, 2001.

20. Ministério da Saúde. Secretaria de Políticas de Saúde. Coordção Nacional de DST e Aids (2001) A Contribuição dos Estudos Multicêntricos frente à epidemia de HIV/Aids entre UDI no Brasil: 10 Anos de Pesquisa e Redução de Danos. Série Avaliação no 8, Brasília, Ministério da Saúde, 2001.

21. Mello MB, Pinho A, Chinaglia MLM, Tun W, Barbosa Júnior A, et al. (2008) Assessment of risk factors for HIV infection among men who have sex with men in the metropolitan area of Campinas city, Brazil, using respondent-driven sampling. 2008. (Relatório de pesquisa). Available http://www.popcouncil.org/ pdfs/horizons/Brazil_MSMRiskFactors.pdf. Accessed 2010 Jun 22.

22. Ministério da Saúde. Secretaria de Vigilância em Saúde. Programa Nacional de DST e Aids (2005) PCAP - Pesquisa de Conhecimento, Atitudes e Práticas na População Brasileira de 15 a 54 anos, 2004. Available http://www.aids.gov.br/main.asp? View $=\% 7$ BE62A8511\%2D7150\%D4615\%2D9BFA\%2D10FDC4F4E642\%7D\&T eam $=\&$ params $=$ sLetraFim $=\mathrm{Q} \% 3 \mathrm{BsLetraIni}=\mathrm{P} \% 3 \mathrm{~B} \&$ UIPartUID $=\% 7 \mathrm{BC} 002 \mathrm{~F} 3$ 20\%2D7F48\%2D4FD5\%2D9036\%2D124927BAF21E\%7D. Accessed 2010 Jun 22.

23. Szwarcwald CL, Souza Júnior PRB (2006) Estimativa da prevalência de HIV na população brasileira de 15 a 49 anos, 2004. In: Boletim Epidemiológico AIDS DST ano III (01):11-15. Available: http://www.aids.gov.br/data/Pages/ LUMIS624DE984PTBRIE.htm. Accessed 2010 Jun 22.

24. Brasil Ministério da Saúde. Secretaria de Vigilância em Saúde. Departamento de DST, Aids e Hepatites Virais (2010) Número de pacientes em terapia anti- retroviral por região e unidade da federação. Brasil, 1999-2008. Available: http:// sistemas.aids.gov.br $/$ monitoraids $/$ ?lang $=$ pt-BR\&pagina $=+\& s t \_l$ Panel $=$ \&indexMe nu $=0 \#$. Accessed 2010 Jun 22.

25. Pilcher CD, Tien HC, Eron JJ, Vernazza PL, Leu SY, et al. (2004) Brief but Efficient: Acute HIV Infection and the Sexual Transmission of HIV. J of Infectious Dis 189: 1785-1792.

26. Powers KA, Poole C, Pettifor AE, Cohen MS (2008) Rethinking the heterosexual infectivity of HIV-1: a systematic review and meta-analysis The Lancet 8(9): 553-63 Epub 2008 Aug4.

27. Vittinghoff E, Douglas J, Judson F, McKirnan D, MacQueen K, et al. (1999) Per-Contact Risk of Human Immunodeficiency Virus Transmission between Male Sexual Partners. Am J Epidemiol 150: 3;306-31.

28. Weller S, Davis K (2004) Condom Effectiveness in Reducing Hetersexual HIV Transmission (Cochrane Review). In Cochrane Library, Issue 1, 2004. Chichester, UK. John Wiley \& Sons, Ltd.

29. International AIDS Vaccine Initiative (2006) The Impact of an AIDS Vaccine in Developing Countries: A New Model and Preliminary Results. Policy Research Working Paper \#8.

30. Barth-Jones DC, Cheng H, Kang LY, Kenya PR, Odera D, et al. (2005) Cost effectiveness and delivery study for future HIV vaccines. AIDS 19 13: w1-6.

31. Barth-Jones DC, Longini Jr. IM (2002) Determining Optimal Vaccination Policy for HIV Vaccines: A Dynamic Simulation Model for the Evaluation of Vaccination Policy. Proceedings of the International Conference on Health Sciences Simulation 2002. Eds. Anderson JG, Katzper, 2002 Western Multiconference, January 27-31, 2002. San Antonio, Texas.

32. Chor D, Lima CRA (2005) Aspectos Epidemiológicos das Desigualdades Raciais em Saúde no Brasil. Cad Saude Publica 21: 1586-94.

33. Jorge MHPM, Gotlieb SLD, Laurenti R (2002) Sistema de Informações sobre Mortalidade: Problemas e Propostas para o seu Enfrentamento I - Mortes por Causas Naturais. Rev Bras Epidemiol 5: 197-211.

34. Blower SM, Dowlatabadi H (1994) Sensitivity and uncertainty and analysis of complex models of disease transmission: an HIV model as an example. Int Statist Rev 2: 229-243.

35. Blower SM, Hartel D, Dowlatabadi H, Anderson RM, May RM (1991) Drugs, sex and HIV: a mathematical model for New York City Philos Trans R Soc Lond B Biol Sci 331(1260): 171-87.

36. Rerks-Ngarm S, Pitisuttithum P, Nitayaphan S, Kaewkungwal J, Chiu J, et al. (2009) Vaccination with ALVAC and AIDSVAX to Prevent HIV-1 Infection in Thailand. N Engl J Med 361: 1-12.

37. Blower SM, McLean AR (1994) Prophylactic vaccines, risk behavior change and the probability of eradicating HIV in San Francisco. Science 265: 1451-1454.

38. Gray RH, Xianbin L, Wawer MJ, Gange SJ, Serwadda D, et al. (2003) Stochastic simulation of the impact of antiretroviral therapy and HIV vaccines on HIV transmission; Rakai, Uganda. AIDS 17: 1941-1951.

39. Nagelkerke NJD, De Vlas SJ (2003) The Epidemiological Impact of an HIV Vaccine on the HIV/AIDS Epidemic in Southern India. Policy Research Working Paper 2978. Washington, DG: World Bank, February.

40. Owens DK, Edwards DM, Shachter RD (1998) Population effects of preventive and therapeutic HIV vaccines in early- and late-state epidemics. AIDS 18: 1057-1066.

41. Smith RJ, Blower SM (2004) Could disease-modifying HIV vaccines cause population-level perversity? Lancet Infect Dis 4: 636-639.

42. Anderson R, Hanson M (2005) Potential public health impact of imperfect HIV type 1 vaccines. J of Infectious Dis 191(Suppl 1): S85-S96.

43. Edwards DM, Shachter RD, Owens DK (1998) A dynamic HIV-transmission model for evaluating the costs and benefits of vaccine programs. Interfaces 28: $144-166$.

44. Blower S, Moss RB, Fernandez-Cruz E (2003) Calculating the potential epidemic-level impact of therapeutic vaccination on the San Francisco HIV epidemic. AIDScience 3: 21. 